\title{
Influence of a Hub-Groove on the Unsteady Losses of a Transonic Compressor Stage
}

\author{
Stefan Rochhausen, Georg Kröger, Eberhard Nicke, Stefan Stollenwerk
}

\begin{abstract}
In this paper the influence of a non-symmetrical sidewall-contour on the flow field of an axial compressor is investigated. In earlier studies, carried out at the DLR Institute of Propulsion Technology in Cologne, it has been shown that it is possible to influence secondary flow regimes by a non-symmetrical sidewall contour. With the help of the contour a vortex is generated that serves as an aerodynamic barrier and thus reduces the flow transport orthogonal to the main flow direction. In this paper the described method of flow manipulation is investigated for the compressor rotor at the hub wall. It turns out that despite the unsteady inflow conditions the vortex is stably generated and works as an aerodynamic separator. The rotor hub region increases its flow capacity. A higher pressure ratio is achieved at a highly loaded operating point. The efficiency at this point remains unchanged.
\end{abstract}

\section{List of Symbols and Abbreviations}

$\frac{m}{M}$ - relative mass flow, $\mathrm{y}^{+}$- dimensionless wall distance, $\eta$ - isentropic efficiency, $\pi$ - total pressure ratio, $\omega$ - vorticity , BPrVar - back pressure variation, MVDR meridional velocity density rate, OP - operating point, PR - pressure ratio, TVD Total Variation Diminishing

\section{Introduction}

In order to meet the ACARE 2020 target of $\mathrm{CO}_{2}$-emission reduction, the implementation of new innovative technologies for gas turbines is necessary. For many decades the blade design has been in the focus of interest for turbomachine com-

Stefan Rochhausen, Georg Kröger, Eberhard Nicke, Stefan Stollenwerk Institute of Propulsion Technology, DLR, Cologne,Germany, e-mail: stefan.rochhausen@dlr.de 
pressor optimisation. Nowadays, with the help of advanced computational fluid dynamics software and high performance computers one is able to gain more detailed insight to the full compressor flow regime. Apart from the blade the two sidewalls at the compressor hub and tip are two more surfaces exposed to the fluid that give the opportunity for aerodynamic optimisation.

In earlier investigations [3],[2],[1] at the DLR Institute of Propulsion Technology it turned out that it is possible to improve the performance of a stator cascade by a non rotation-symmetric sidewall contour. In this work it has been shown, that secondary flows can be suppressed by using an auxiliary vortex. The auxiliary vortex is working as an aerodynamic barrier. It reduces the transport of fluid at the hub wall. Especially in off-design points significant improvements in the flow field have been achieved. In the present paper the influence of a non rotation-symmetric hub wall on the flow field of a compressor rotor is discussed. The main difference is that the rotor is subject to the unsteady inflow conditions. The effect of pressure fluctuations and velocity gradients on the vortex generation should be examined. The aim is mainly to examine the vortex-producing mechanism rather than carrying out a highly sophisticated rotor-optimisation.

\section{The Numerical Method}

The program applied in this work is the TRACE-Code (Turbomachinery Research Aerodynamics Computational Environment) which is developed by the DLR's Institute of Propulsion Technology in Cologne [11] [6]. TRACE is a Reynolds-Averaged Navier-Stokes solver for multiblock meshes using a cell-centered finite-volume approach. Convective fluxes are discretised using a TVD upwind scheme by Roe, for the viscous fluxes central differences are used. Time accurate non-reflecting inflow and outflow boundary conditions are implemented [8] [5] [4]. For turbulence modeling the Wilcox-k- $\omega$-model in combination with turbomachinery extensions is used [12]. The phase-lag method according to $\mathrm{He}$ [10] is used for simulating real blade count ratios.

\section{The Research Object}

This investigation has been carried out for the DLR-Rig250. This rig is a 4.5-stage transonic research compressor with a pressure ratio of about 4.54. For this work a configuration containing the stators of stage 2 and 3 as well as the rotor of stage 3 have been simulated. Stator 2 and rotor 3 are simulated using the phase lag methodology whereas the coupling between rotor 3 and stator 3 is modeled by a mixing plane. The full model consists out of 29 structured blocks with overall number of approximately $1.87 \mathrm{M}$ cells. In radial direction the mesh has 51 nodes, thus a wall law boundary layer formulation is applied at the hub and tip walls. For the blade 
surfaces a low-Reynolds mesh is generated with a $y^{+}=O(1)$. A side view of the full numerical model is provided in figure 7 .

\section{Global Results}

The inflow and outflow boundary conditions for the operating point OP B have been taken from earlier full machine simulations. In the present work a working line has been simulated by modifying the backpressure at the outflow boundary whilst the inflow boundary has been fixed. The rotational speed is constant in all operating points. The backpressure has been raised in small steps until no convergence has been achieved for the flow solution. In this study, for the convergence criterion, the mass flow rate over the outflow boundary has been defined. Simulations with a mass flow change of less than $0.2 \%$ in 1000 time steps are considered as converged.

Here four operating points are presented. Operating point OP A is the point with the highest backpressure for which numerical convergence has been achieved. For all operating points the backpressure variations corresponding to OP B are given in table 1 . Also in table 1 the basic globally averaged performance results are presented.

In figure 1 the total pressure ratio (PR) for the rotor 3 is depicted over the mass flow. This compressor map shows that the compressor with the contoured sidewall achieves slightly higher pressure ratios for all operating points. In operating point OP A, the closest point to the "numerical surge line", the difference is clearly visible. In this example the sidewall contour increases the pressure ratio at highly loaded operating points, close to the surge line. In figure 2 the efficiency ${ }^{1}$ map for rotor 3 is provided. For OP A and OP B the efficiency difference between the basis rotor and the contoured rotor is negligible. For OP C and OP D the contoured rotor has a lower efficiency. In the following sections the focus is first set on the generation mechanism of the vortex and second on the flow regime at the highly loaded operating point OP A.

Table 1 Global Results of the working line calculations (relative to OP C)

\begin{tabular}{lllll}
\hline Operating Point & BPrVar & Massflow & PR & Efficiency \\
\hline OP D basis & $-10.0 \%$ & $+9.22 \%$ & $-5.55 \%$ & $+1.52 \%$ \\
OP D contour & $-10.0 \%$ & $+9.17 \%$ & $-5.55 \%$ & $+1.39 \%$ \\
OP C basis & $-5.00 \%$ & $+6.13 \%$ & $-3.00 \%$ & $+1.44 \%$ \\
OP C contour & $-5.00 \%$ & $+6.18 \%$ & $-3.00 \%$ & $+1.36 \%$ \\
OP B basis & $0.00 \%$ & $0.00 \%$ & $0.00 \%$ & $0.00 \%$ \\
OP B contour & $0.00 \%$ & $0.00 \%$ & $0.00 \%$ & $0.00 \%$ \\
OP A basis & $+2.00 \%$ & $-3.28 \%$ & $+0.59 \%$ & $-1.70 \%$ \\
OP A contour & $+2.00 \%$ & $-3.64 \%$ & $+0.72 \%$ & $-1.70 \%$ \\
\hline
\end{tabular}

\footnotetext{
${ }^{1}$ using total values in the rotational coordinate system
} 


\section{Sidewall Contour Design}

The initial idea for this work is to generate a vortex structure with the help of the sidewall in order to reduce the secondary flow structures in the compressor rotor. Therefore the sidewall-contour design must meet the two requirements: Generating a vortex and guiding it through the blade passage in order to suppress secondary flows.

The outcome of the optimisation investigations carried out by Dorfner

[3],[2] was a groove that ranges from the leading edge through the blade passage. For the present study a similar design has been chosen. In figure 6 the shape of the hub wall is depicted in a top view perspective. The groove can be subdivided into two regions according to the two functions: the first part, the vortex generation part, is comparable to a backward facing step. The second part forces the vortex into a direction parallel to the main stream direction, the vortex guidance-edge.

The modification of the sidewall contour has been made without changing the annulus area to achieve comparability to the basis rotor. During the design process it turned out, that a certain minimum length of the vortex generation edge is essentially important to generate a stable vortex. In this early design study a length about $10 \%$ of a blade pitch has been chosen. A more detailed investigation of the contour design can be found in Rochhausen [9].

\section{Vortex Generation}

At first, figures presenting the flux-averaged flow field of the unsteady data-set are discussed. In figure 8 a streamline plot of the rotor hub-wall is shown. The mentioned two different regions can be identified. In the first region the streamlines converge to a separation line. According to Murray and Tobak [7] converging streamlines on a sidewall is an indication of a three-dimensional flow separation. This phenomenon can be explained as follows: The distance between two streamlines can be described as the width of a $3 \mathrm{~d}$-streamtube. If that distance decreases in flow direction, the streamtube gradually increases its height ${ }^{2}$. At that point, at which all streamlines converge to a single line, the $3 \mathrm{~d}$-streamtube fully separates from the surface. Furthermore in figure 8 the second region, the deflection-region of the contour, is visible.

With the help of the next figures the vortex generation at the separation edge should be examined. At this place it is helpful to provide a vortex definition. The rotation of a flow field is defined as the curl of the velocity field. It can be calculated by the equation

$$
\omega=|\nabla \times \mathbf{V}|
$$

\footnotetext{
${ }^{2}$ provided that the continuity equation is applicable
} 
A local maximum of the rotation can be used to identify a vortex core. This relatively simple definition is suitable for identifying vortices in a homogenous flow field. It is not applicable to rotationally flows like boundary layer or shear flows.

Figure 9 shows a plane orthogonal to the separation line. In the plane the vorticity of the flow field is visualised. Close to the separation line a maximum of vorticity is visible that marks the core of the vortex. In figure 10 the vortex structure is visualised by $3 \mathrm{~d}$-streamlines passing through this region of high vorticity. The streamlines are highly twisted and thus they clearly prove the existence of a vortex. At this point it can be stated that a vortex is existing in the flux averaged solution of the unsteady data set.

The time accurate influence of the unsteady flow regime on the vortex generation is shown in figure 11. Here streamlines on the endwall are depicted together with a plane that shows vorticity levels. In the unsteady simulations a full blade passage is represented by 64 time steps, for every fourth time step a flow solution output is written. Accordingly 16 timesteps show a full blade passage. Most of these timesteps differ not much from each other. To focus on the impact of the stator wake the following analysis is exemplified on a few time steps only. The figures of the time steps $1-8$ and $14-16$ differ not much from the situation in time step 9 of figure 11 . By the time the stator-wake reaches the separation line, a significant reduction in the peak vorticity is visible. It can be seen in the time steps number 11, 12 and 13. At this time the angle by which the streamlines hit the separation line is lower. Thus a less strong vortex is generated. It is clearly visible that the wake of the upstream stator has a significant impact on the vortex generation for a short period of time. The duration of this influence is in the order of one eights of a blade passage (approx. 2 out of 16 timesteps). Since the duration of the influence is that small the time averaged figures show that the vortex generation mechanism is stable in the unsteady flow regime.

\section{Radial Distributions}

In the previous chapter the existence of a vortex has been proved. Here the impact of the vortex on the flow field should be discussed based on time-averaged data (Fig. 3-5). The operating point is OP A - the point with the highest backpressure. On the $y$-axis the normalised mass flow is shown. The basis-rotor is represented by the continuous line, the contoured rotor by a dashed line. In figure 3 the radial distribution of the MVDR is shown. The MVDR can be interpreted as a mass flow ratio per area between rotor inlet and outlet. The radial distribution of the MVDR shows two important tendencies. The contoured rotor has a lower MVDR at the close-to-hub region $\frac{m}{M} \approx 0-5 \%$. Above this region, between $\frac{m}{M} \approx 5-30 \%$, the MVDR of the contoured rotor is higher. The reason for this is the effect of the vortex. On the one side the vortex displaces fluid in the close-to-hub region. On the other side it suppresses the secondary flow, and thus more fluid can pass in the region above. The differences in the MVDR $\frac{m}{M}>30 \%$ are relatively small. 
In figure 4 the total pressure ratio $\Pi_{t o t}$ for the rotor is provided. The contoured rotor delivers a slightly higher pressure ratio. This already became evident from figure 1. Figure 4 shows, that greatest difference in pressure ratio can be found in the region $\frac{m}{M} \approx 5-35 \%$, which is approximately the region of the greatest increase in MVDR. The third radial distribution, the isentropic efficiency in figure 5, shows another effect of higher pressure ratio and higher MVDR. In the region $\frac{m}{M} \approx 5-$ $30 \%$, the efficiency of the contoured rotor is up to $2 \%$ higher. Furthermore the figure shows, that the contoured rotor has a lower efficiency in the tip region.

Based on these observations the following conclusions can be drawn: As shown in the figures $8-10$ a vortex is generated. In a region close to the hub $\left(\frac{m}{M} \approx 5-\right.$ $30 \%$ ) this vortex increases the amount of fluid passing the rotor. The rotor produces a higher pressure ratio and thus the efficiency in this region is better. Finally the rotor can not benefit from the higher efficiency. The radial redistribution of the flow changes the entire flow field in the tip region in a way that efficiency in the tip region is reduced.

\section{Summary}

By contouring the sidewall a vortex can be generated which works like an aerodynamic barrier. This effect has been observed in the unsteady and the unsteady averaged flow solutions. It can be concluded, that the influence of the unsteady inflow conditions does not prevent the separation effect. The separation effect increases the flow capacity at the rotor hub. As a consequence the aerodynamic load of the rotor is reduced; the modified rotor shows a better efficiency in the hub region. Furthermore the pressure ratio in the hub region is higher.

In this example the desired capability to achieve higher flow rates at the rotor hub is associated with a change of the flow regime at the rotor tip. The overall performance of the rotor is not significantly improved. Nevertheless it has been shown that in high-performance compressors the sidewalls offer an additional surface for further improvements. In future compressor designs an optimisation incorporating sidewalls might offer several new opportunities. The full annulus must be optimised coinstantaneously since local changes in the geometry influence the flow field globally.

\section{References}

1. W. Steinert E. Nicke H. A. Schreiber A. Hergt, C. Dorfner, Advanced non-axisymmetric endwall contouring for axial compressors by generating an aerodynamic separator part 2: Experimental and numerical cascade investigation, ASME Journal of Turbomachinery 133 (2011), 02102710210278.

2. E. Nicke R. Mönig C. Dorfner, A. Hergt, Advanced non-axisymmetric endwall contouring for axial compressors by generating an aerodynamic separator part 1: Principal cascade 
design and compressor application, ASME Journal of Turbomachinery 133 (2011), 0210261 0210266.

3. Christian Dorfner, Entwicklung eines verfahrens zur konstruktion nichtrotationssymmetrischer seitenwandkonturen in axialen verdichtern, Ph.D. thesis, DLR, Institut für Antriebstechnik, Köln, 2009.

4. D. Kozulovic H. Yang, T. Röber, Hybrid-grid simulation of unsteady wake-boundary layer interaction on a high lift low pressure turbine airfoil, ASME-Paper, GT2003-3842, 2007.

5. E. Nicke A. Weber H. Yang, D. Nürnberger, Numerical investigation of casing treatment mechanisms with conservative mixed-cell approach, ASME-Paper, GT2003-3842, 2003.

6. D. Nürnberger M. Franke, E. Kügeler, Dlr-verfahren trace: Moderne simulationstechniken für turbomaschinenströmungen, DLR, Cologne Germany, Deutscher Luft- und Raumfahrtkongress JT2005-211, 2005.

7. David J. Peake Murray Tobak, Topology of three-dimensional seperated flows, Nasa tm-81294, NASA, Ames Research Center, Moffett Field, California, 1981.

8. D. Nürnberger, Implizite zeitintegration für die simulation von turbomaschinenströmungen, Ph.D. thesis, Ruhr-Universität Bochum, Bochum also DLR FB 2004-27., 2004.

9. S. Rochhausen, Untersuchung des einflusses verschiedener nabenkonturierungen auf das instationäre verlustverhalten einer 1,5 -stufigen transsonischen verdichterkonfiguration, Diploma Thesis also DLR IB 325-04-10, TU Braunschweig, Braunschweig, Germany, 2010, 2010.

10. R. Schnell, Numerische simulation des akustischen nahfelds einer triebwerksgebläsestufe, Ph.D. thesis, Technische Universität Berlin, Berlin, Germany also DLR FB 2004-23, 2004.

11. A. Weber, Trace user's manual, DLR, Cologne Germany, IB 325-07-06, 2006.

12. David C. Wilcox, Turbulence modeling for cfd, DCW Industries, 2000.

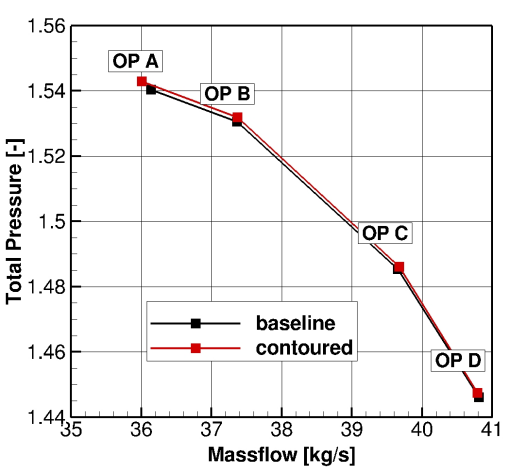

Fig. 1 Compressor Map

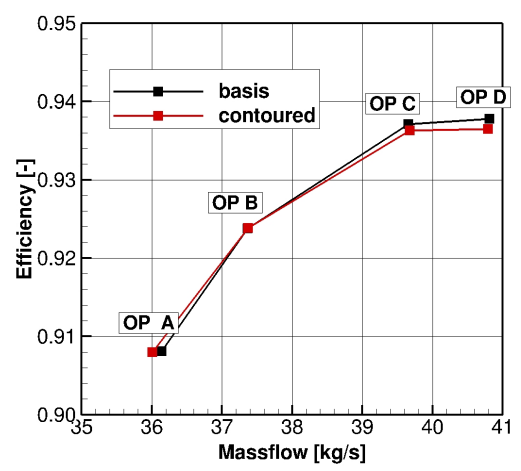

Fig. 2 Isentropic Efficiency 


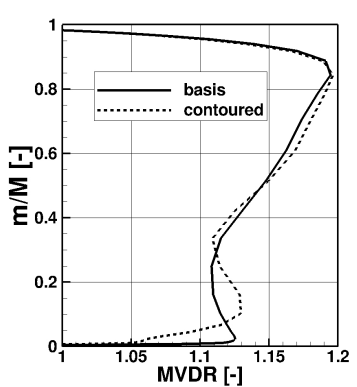

Fig. 3 MVDR

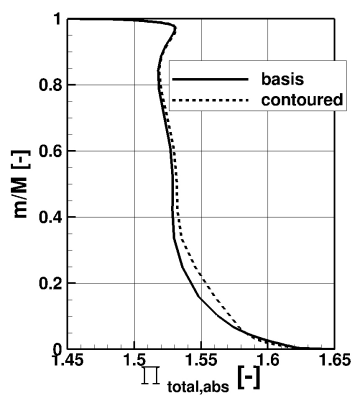

Fig. 4 Total Pressure Ratio

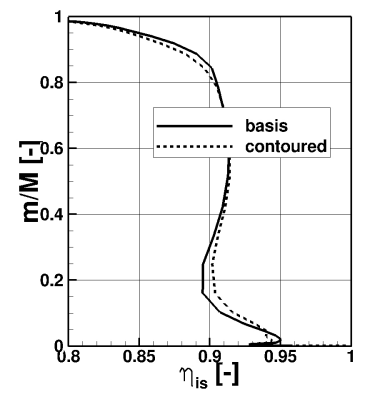

Fig. 5 Isentropic Efficiency

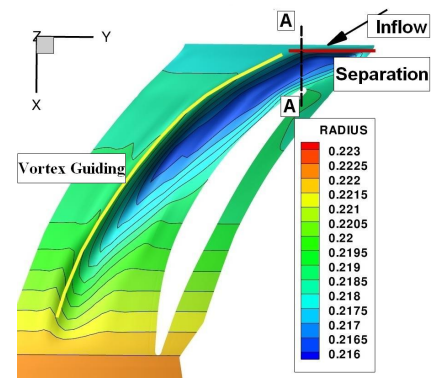

Fig. 6 Top View of the Contour

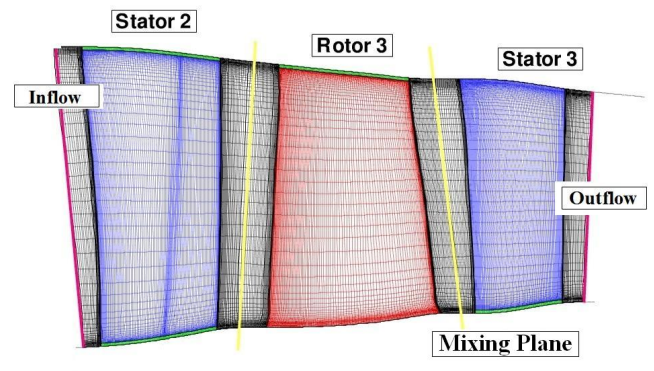

Fig. 7 The Simulation Domain

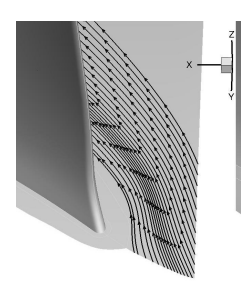

Basis Rotor

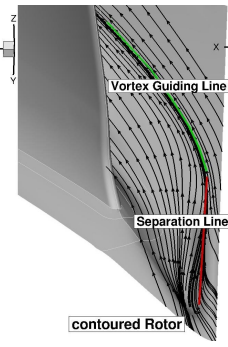

the Endwall

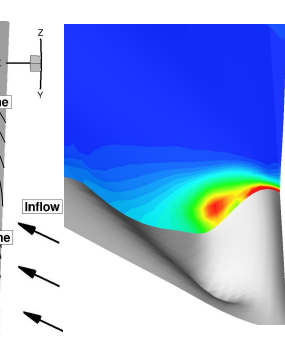

Fig. 9 Vorticity: Y const. Plane

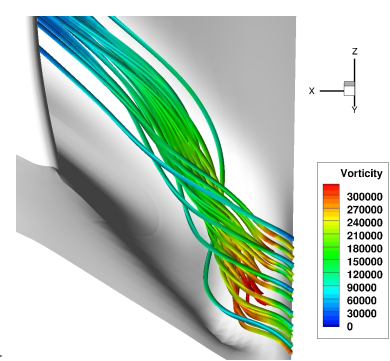

Fig. 10 3d-Streamlines
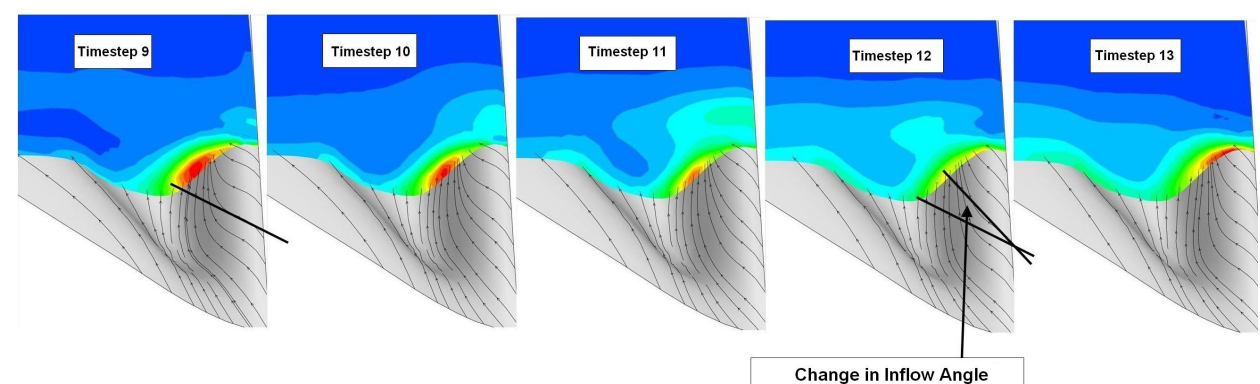

Change in Inflow Angle

Fig. 11 Movieplot: Wake Passing the Separation Edge 